\title{
Finite volume method for simulations of traffic dynamics with exits and entrances
}

\author{
S. R. Pudjaprasetya ${ }^{1} \quad$ P. Z. Kamalia ${ }^{2}$
}

(Received 14 September 2017; revised 9 May 2018)

\begin{abstract}
We discuss a macroscopic study of vehicle traffic dynamics in which traffic flow is considered as a continuum governed by the kinematic Lighthill-Whitham-Richard model, with a source term accounting for traffic entries and exits through a junction. The kinematic model is solved using finite volume method, with the flux function is approximated using the upwind method. In order to prevent flows which exceed road capacity and to preserve the positivity of traffic density in simulations with entries and exits, a stop-go procedure is adopted. The resulting scheme is used to simulate the responses of traffic density to light and heavy entrances from a junction, and the dynamics predicted by the simulations are shown to conform well to analytical solutions. With a validated numerical algorithm at hand, we simulate traffic dynamics for several scenarios on a roadway with entrances and exits. Depending on the rate of exit or entrance and the initial condition,
\end{abstract}

DOI:10.21914/anziamj.v60i0.12435, (c) Austral. Mathematical Soc. 2018. Published 2018-08-19. ISSN 1445-8810. (Print two pages per sheet of paper.) Copies of this article must not be made otherwise available on the internet; instead link directly to the DOI for this article. 
density profiles in the form of shock wave and rarefaction wave may appear.

Subject class: 35L65, 90B20

Keywords: Hyperbolic Conservation Laws, Continuum Traffic Model, finite volume method

\section{Contents}

1 Introduction

E2

2 Mathematical model and its discrete formulation

E4

3 Validation of numerical simulation with exit and entrance

E7

4 Impact of exits and entrances to the main road

E11

4.1 More experiments with exits and entrances . . . . . . . .

E18

5 Conclusions

E21

References

E23

\section{Introduction}

In urban areas, a vehicle traffic system consists primarily of a network of roadways through which traffic flows in and out via junctions. Traffic congestion can occur when vehicles moving along a road segment approach a junction. The effect of an inflow of vehicles at an entry point along a road is to introduce a local congestion that may then propagate upstream, whereas the presence of an exit can help to relieve congestion, leading to a local increase in traffic speed. Using a standard continuum model, we develop a numerical 
model to simulate such situations, with potential applications in road network management and design.

Although traffic simulation packages have been in use since the 1960s [11], modelling and numerical simulation of traffic remain an active area of study, with various open problems continuing to attract the attention of researchers. The accurate simulation of traffic inflow and outflow via junctions is one such question. One widely used numerical model is the cell transmission model. This method, based on a supply-demand principle, was first proposed by Daganzo [2] and Lebacque [7], and reviewed by Orosz et al. [10]. Kinematic wave models of merging traffic have also been studied using this method by Daganzo [3], Holden and Risebro [6], and Lebacque [7]. Nowadays, for macro-scale modelling of traffic dynamics, the most commonly adopted model is the kinematic Lighthill-Whitham-Richards (LWR) model. This model uses a conservation law to describe traffic on a road segment in terms of traffic density and flux. In-depth discussions of the LWR model are available in several textbooks $[5,4,8]$. An analytical study of traffic dynamics at junctions was presented by Mercier [9], where the interactions between several lanes of traffic were analyzed. Bagnerini et al. [1] studied the dynamics of traffic flowing into and out of junctions using a systems of equations analogous to a fluid dynamics model. In the current study, traffic flows into and out of road segments via entrance and exit areas which are introduced into the model by the inclusion of a source term in the kinematic LWR model.

Aiming to enhance the capability of this method to accurately implement the LWR model with a source term, we adopt a variant of the finite volume method that uses an upwind approximation for the flux function. Section 2 formulates the kinematic LWR model with a Greenshield flux function, followed by a description of its consistent discretization. The decision to adopt the Greenshield flux here is motivated by the availability and simplicity of its analytical solution; however, the numerical method discussed in this paper can directly be modified in a straightforward manner to handle other concave flux functions commonly used for traffic modelling such as the Greenberg, Underwood, Northwestern, and Pipes-Munjal functions. Section 3 compares 
numerical results obtained using this scheme with analytical solutions. Finally, in Section 4, we present examples of simulated traffic dynamics under several scenarios involving exits and entrances.

\section{Mathematical model and its discrete formulation}

We consider heavy traffic moving in one direction, say the $x$-direction. Letting $n(x, t)$ (vehicles $/ \mathrm{km}$ ) represent the traffic density as a function of the spatial variable $x$ and time $t$, and invoking a conservation principle leads to the kinetic LWR equation

$$
n_{t}+f(n)_{x}=\beta,
$$

in which $f$ (vehicles/hour) denotes the flux of traffic, which is the number of vehicles passing through a certain position per unit of time. The source term $\beta(x, t)$ (vehicles/(km hour)) denotes the net number of vehicles per $\mathrm{km}$ per hour entering or leaving at a certain position.

In traffic dynamics modelling, traffic flux is typically assumed to depend on traffic density. This dependence is often approximated using the Greenshield traffic flux function

$$
\mathrm{f}(\mathrm{n})=n V_{m}\left(1-\frac{n}{N_{m}}\right),
$$

where $V_{m}(\mathrm{~km} /$ hour $)$ denotes the maximum velocity of vehicles in empty roads, and $N_{m}$ (vehicles $/ \mathrm{km}$ ) denotes the maximum capacity of single-lane traffic. The curves $V(n)$ and $f(n)$ are plotted in Figure 1. While the Greenshield model may seem simplistic, it nonetheless succeeds in capturing the important features of traffic flow: flux is zero when traffic density is either zero $(n=0)$ or at maximum capacity $\left(n=N_{m}\right)$, and the flux attains its maximum for an intermediate value of traffic density, $n=N_{m} / 2$.

A discrete formulation of (1) on a computational domain $[0, X]$ is formulated in this paragraph. Let the computational domain be divided into $\mathrm{N}$ cells of 
Figure 1: (top) the Greenshield velocity $v(n)$, and (bottom) the corresponding flux $f(n)$.
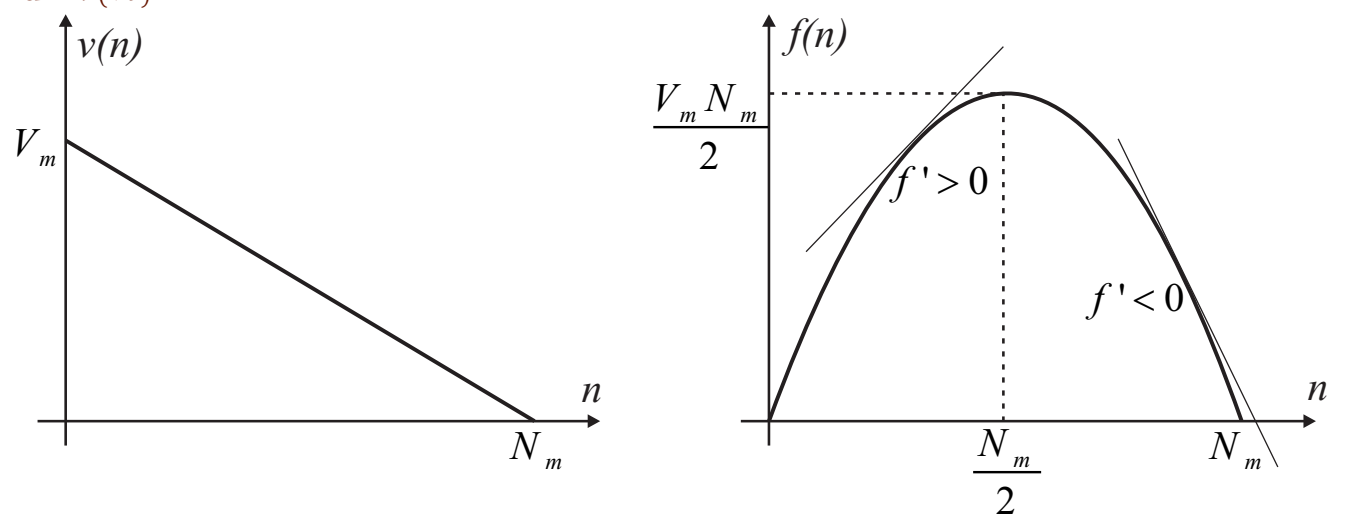

length $\Delta x$ in a staggered manner, with partition points $x_{1 / 2}=0, x_{3 / 2}=\Delta x$, $\ldots, x_{i+1 / 2}=i \Delta x, \ldots, x_{N+1 / 2}=N \Delta x=X$. On the cell $C_{i}=\left[x_{i-1 / 2}, x_{i+1 / 2}\right]$, the finite volume discretization of (1) reads

$$
n_{i}^{j+1}=n_{i}^{j}-\frac{\Delta t}{\Delta x}\left({ }^{*}{ }_{i+1 / 2}^{j}-{ }^{*} f_{i-1 / 2}^{j}\right)+\beta_{i}^{j} \Delta t .
$$

Equation (3) states that at every time step $t_{j}$, the value of $n_{i}$ is to be updated using the net flux from left and right boundaries, as well as any internal entrance/exit sources $\beta_{i}^{j} \Delta t$. Here, we implement a first-order upwind approximation for flux ${ }^{*} f_{i+1 / 2}^{j}$ : for any time step $t_{j}$, the signal speed at the staggered point $x_{i+1 / 2}$ is approximated by $\frac{1}{2}\left(f_{i}^{\prime}+f_{i+1}^{\prime}\right)$, and its sign is used in the upwind approximation

$$
{ }^{*} f_{i+\frac{1}{2}} \approx \begin{cases}f_{i} & \text { if } \frac{1}{2}\left(f_{i}^{\prime}+f_{i+1}^{\prime}\right)>0 \\ f_{i+1} & \text { if } \frac{1}{2}\left(f_{i}^{\prime}+f_{i+1}^{\prime}\right)<0\end{cases}
$$

In this upwind approximation, when the signal speed is positive then $\mathrm{f}_{i+1 / 2}^{j} \approx$ $f_{i}^{j}=n_{i}^{j} V_{m}\left(1-n_{i}^{j} / N_{m}\right)$, whereas when the signal speed is negative then $f_{i+1 / 2}^{j} \approx f_{i+1}^{j}=n_{i+1}^{j} V_{m}\left(1-n_{i+1}^{j} / N_{m}\right)$, see Figure 2. The value of $n_{i}$ is 
Figure 2: Upwind approximation for ${ }^{*} f_{i+1 / 2}$; this holds for every time iteration.
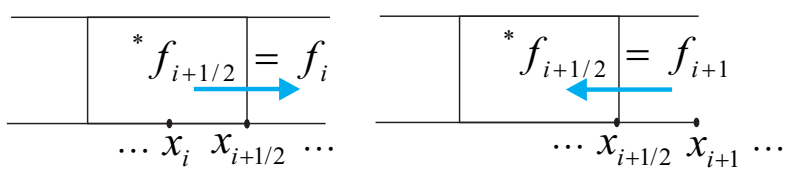

assumed to be constant in cell $C_{i}$. The above scheme is stable under the CFL-like condition $V_{m} \Delta t / \Delta x \leqslant 1$. Moreover, as discussed by Mattheij et al. [8], this scheme can accurately simulate Riemann solutions, including the propagation of shock waves with the correct speed. Previously [12], the scheme was used to analyse the performance of traffic lights in comparison with roundabouts.

In cases where entrances and exits bring about local increases and reductions in traffic density, in order to ensure that traffic density values $n(x, t)$ remain between zero and the maximum density $\mathrm{N}_{\mathrm{m}}$, a kind of stop and go procedure is implemented as follows. For the case $\beta_{i}^{j}>0$

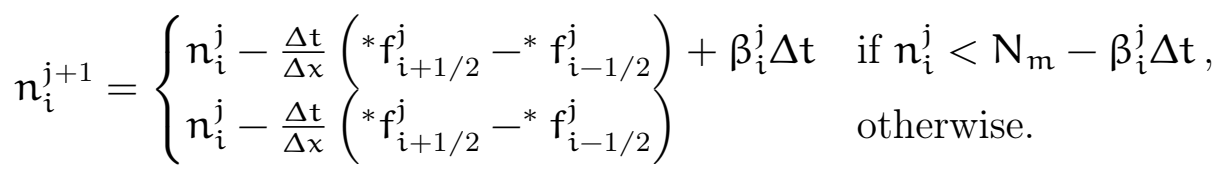

For the case $\beta_{i}^{j}<0$

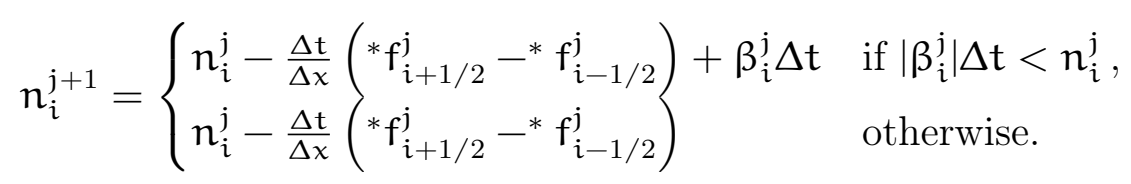

Interpretation of (5) is that the cell $C_{i}$ may receive an entry $\beta_{i}^{j} \Delta t$ with $\beta_{i}^{j}>0$, if its current density $n_{i}^{j}$ is still below the maximum $N_{m}$, or more precisely $n_{i}^{j}<N_{m}-\beta_{i}^{j} \Delta t$. On the other hand, formula (6) means that vehicles may exit from cell $C_{i}$ provided that its current density is such that $n_{i}^{j}>\left|\beta_{i}^{j}\right| \Delta t$. 


\section{Validation of numerical simulation with exit and entrance}

In this section, we first discuss the analytical solution that is used to validate our numerical results. Applying the method of characteristics to (1), we find that along the characteristic $\frac{d x}{d t}=f^{\prime}(n)$, the value $n(x, t)$ changes according to $\frac{\mathrm{d} n}{\mathrm{dt}}=\beta$. When $\beta(x, t) \stackrel{\mathrm{dt}}{=} \beta_{0}$ is constant, the characteristic method yield an explicit formula. Detailed descriptions of this approach are widely available [5], but we review the method here for clarity. Considering the method of characteristics applied to (1) with initial condition $\mathfrak{n}(x, 0)=\mathfrak{n}_{0}$. Along the characteristic curve, the value of $n$ grows linearly in time as $n(x(t), t)=\beta_{0} t+n_{0}$. Moreover, given this explicit function of $n$, the differential equation for the characteristic curve reads

$$
\frac{d x}{d t}=V_{m}\left(1-\frac{2 \beta_{0} t+2 n_{0}}{N_{m}}\right)
$$

which is solved to yield the characteristic parabola

$$
x(t)=V_{m}\left(1-\frac{2 n_{0}}{N_{m}}\right) t-\beta_{0} \frac{V_{m}}{N_{m}} t^{2}+C .
$$

We consider a roadway of length $X$, of which the left section is a junction from which vehicles may enter or exit the roadway. The junction extends from position 0 until $X_{E}$, with $X_{E}<X$. First, we assume that exits and entrances occur at a constant rate $\beta_{0}$. The mathematical model for this case is PDE (1) with

$$
\beta= \begin{cases}\beta_{0}, & \text { for } 0<x<X_{E}, \\ 0, & \text { for } x>X_{E} .\end{cases}
$$

Next, we study how traffic density evolves as a result of entrances along $0<x<X_{E}$. For this purpose, we take an empty roadway state $n(x, 0)=0$ as the initial condition. 
Figure 3: A sketch of characteristic curves for the low entrance rate case, $0<\beta_{0}<\mathrm{V}_{\mathrm{m}} \mathrm{N}_{\mathrm{m}} / 4 \mathrm{X}_{\mathrm{E}}$.

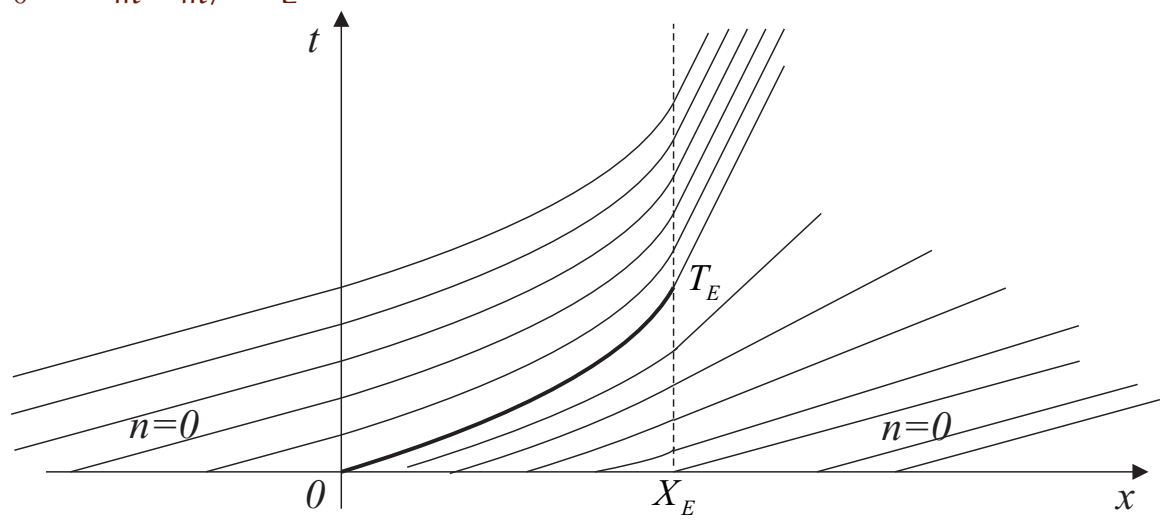

Initially, areas $x \leqslant 0$ and $x \geqslant X_{E}$ are quiet zones, in these areas, the characteristic are straight lines with gradient $d x / d t=V_{m}$, see Figure 3 . In the entrance area, the characteristic curves emanating from $(\xi, 0)$, with $0<\xi<X_{E}$, are parabolas

$$
x(t)=V_{m} t-\beta_{0} \frac{V_{m}}{N_{m}} t^{2}+\xi .
$$

Along this parabola, traffic density increases linearly in time as $n=\beta_{0} t$. After leaving the entrance region, the characteristic curves become straight lines with gradient $f^{\prime}\left(\beta_{0} t\right)$. Let the parabola emanating from $x=0$ at time $\mathrm{t}=0$ be denoted as

$$
x_{\mathrm{O}}(\mathrm{t})=\mathrm{V}_{\mathrm{m}} \mathrm{t}-\beta_{0} \frac{\mathrm{V}_{\mathrm{m}}}{\mathrm{N}_{\mathrm{m}}} \mathrm{t}^{2} .
$$

This parabola crosses $x=X_{E}$ at time $t=T_{E}$, with

$$
\mathrm{T}_{\mathrm{E}}=\left(1-\sqrt{\frac{1-4 \beta_{0} X_{\mathrm{E}}}{N_{\mathrm{m}} \mathrm{V}_{\mathrm{m}}}}\right) \frac{\mathrm{N}_{\mathrm{m}}}{2 \beta_{0}} .
$$

Characteristics emanating from $(0, \tau)$, with $\tau>0$, are parabolas

$$
x(t)=V_{m}(t-\tau)-\beta_{0} \frac{V_{m}}{N_{m}}(t-\tau)^{2} .
$$


Together with the parabola $x_{\mathrm{O}}(t)$, these parabolas straighten out to become lines with gradient $f^{\prime}\left(\beta_{0} T_{E}\right)$. Figure 3 plots the characteristic curves in the $x t$-plane.

Once the characteristics are obtained, the corresponding analytical solutions are then constructed. Along the parabolae, traffic density increases linearly in time, whereas along the straight continuation lines, traffic density is constant. As an example, along the parabola $x_{\mathrm{O}}(t)$ the zero initial traffic density grows linearly in time until time $T_{E}$, at which point traffic density reaches the value $n\left(T_{E}\right)=\beta_{0} T_{E}$. After that, for times $t>T_{E}$, traffic density remains constant along the straight characteristic lines with gradient $d x / d t=f^{\prime}\left(\beta_{0} t\right)$. Here we simulate two conditions: one with a relatively light influx of entrances; and another with a relatively heavy influx of entrances.

Light entrance case We construct a computational domain over the interval $[0, X=30]$, with an entrance region spanning $\left[0, X_{E}=5\right]$. For the simulation, we use normalized parameters $\mathrm{N}_{\mathrm{m}}=1, \mathrm{~V}_{\mathrm{m}}=1$, with $\Delta \mathrm{x}=\Delta \mathrm{t}=0.005$. In the entrance region, traffic enters at a constant rate $\beta_{0}$, and (3) is implemented using $\beta_{i}^{j}=0.04$. The resulting traffic densities computed for subsequent times are plotted in Figure 4 (top). The analytical solutions are shown alongside these numerical results for validation.

Beginning directly from the outset, traffic density in the entrance region $\left[0, X_{E}\right]$ increases. As time progresses, it continues to increase until finally traffic enters the downstream area $x>X_{E}$ in the form of a rarefaction wave. The time at which the characteristic crosses $X_{E}=5, T_{E}=6.9098$, agrees with the value from the analytical formula (11). Figure 4 (bottom) displays the contour plot of the calculated traffic density and the characteristic parabola $x_{O}(t)$. Here, the parabola $x_{O}(t)$ visibly crosses through the contours precisely at their corners, which again confirms the calculated traffic density profile $\mathfrak{n}(x, t)$. As time progresses, we also calculate the total traffic volume of the computed traffic density, defined as $\operatorname{Vol}\left(t_{j}\right) \equiv \sum_{i=1}^{N} \mathfrak{n}\left(x_{i}, t_{j}\right) \Delta x$, and it confirms the 
Figure 4: Simulation of the light entrance case: (top) traffic density at subsequent times $\mathrm{t}=3,5, \mathrm{~T}_{\mathrm{E}}, 10,15$ from numeric (solid lines) and analytic (crosses); (below) contour plots of numerical traffic density together with the characteristic parabola $x_{\mathrm{O}}(\mathrm{t})$.
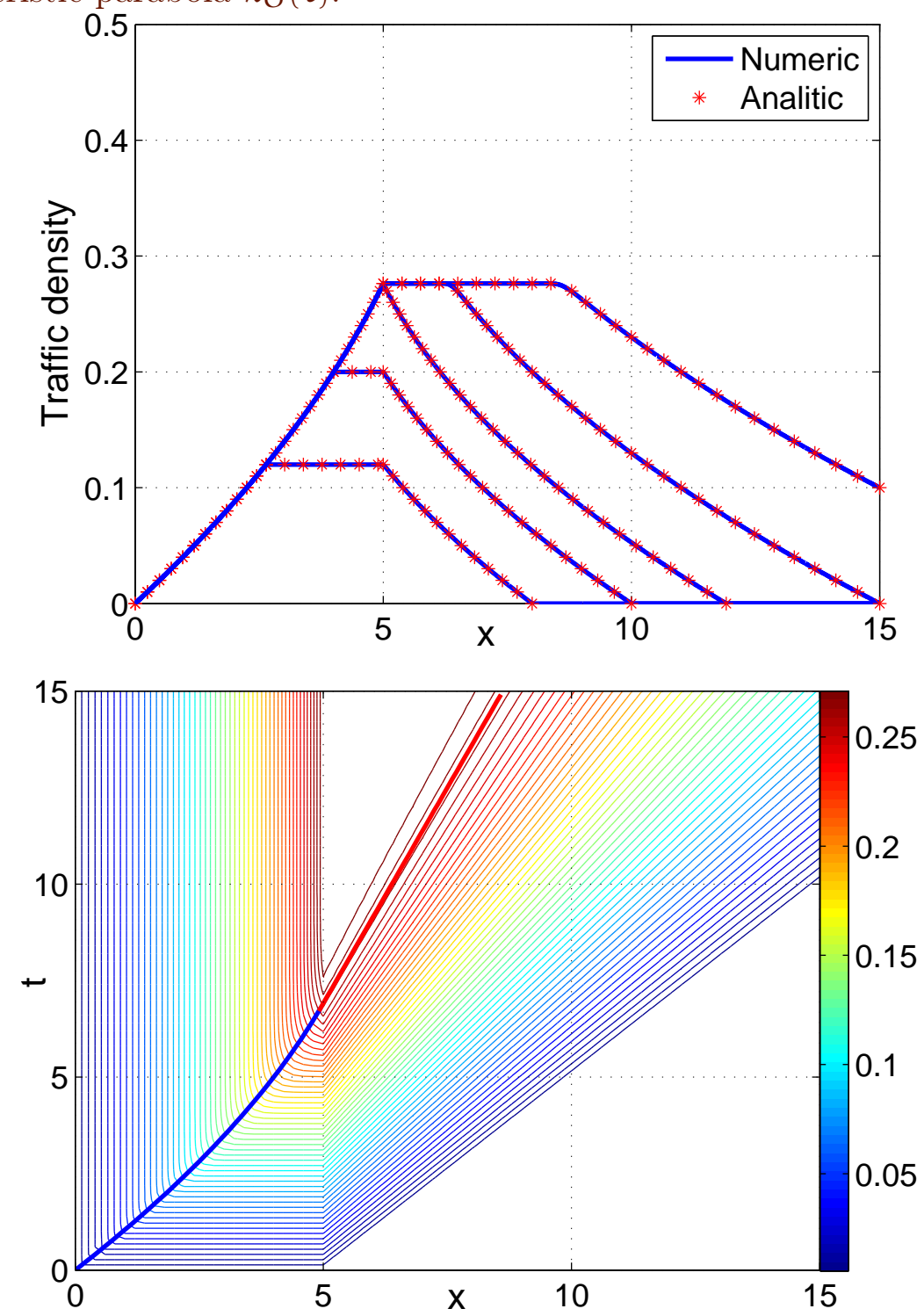
analytical formula

$$
\operatorname{Vol}(t)= \begin{cases}\beta_{0} X_{E} t & 0<t<T_{E} \\ \beta_{0} X_{E} T_{E} & t>T_{E} .\end{cases}
$$

Heavy entrance case In this second simulation, we use the same parameter values as in the previous simulation $\left(\mathrm{V}_{\mathrm{m}}=1, \mathrm{~N}_{\mathrm{m}}=1\right.$, and $\left.\mathrm{X}_{\mathrm{E}}=5\right)$, but now we consider a somewhat heavier entrance rate of $\beta_{i}^{j}=0.08$. Figure 5 presents the calculated traffic density. As time progresses, the traffic density continuously builds up until it reaches half of the maximum density $\mathrm{N}_{\mathrm{m}} / 2$, which then induces a backward-propagating signal. For the simulation displayed in Figure 5, the stop-go procedure plays an important role in preventing traffic densities from increasing beyond the maximum capacity $\mathrm{N}_{\mathrm{m}}$.

\section{Impact of exits and entrances to the main road}

The previous section validates the scheme for scenarios involving light and heavy entrance rates. In this section we conduct simulations on a rather long road segment with a section containing an exit-entrance region, and we are interested on traffic density after a long simulation time. We investigate two distinct types of exit-entrance influx: constant rate and Poisson-distributed rate.

Consider a main road with traffic running to the right. As time progresses, traffic density evolves in response to exits and entrances from the junction. Here, we implement the scheme (3) on a computational domain $[-X, X]$, with a junction or exit-entrance area located on the interval $\left[0, X_{E}\right]$ ilustrated in Figure 6. In all figures in this section, exits-entrances areas are expressed in green. 
Figure 5: Simulation of the heavy entrance case at time $t=30$ : (top) numerical traffic density at subsequent times; and (bottom) their contours.
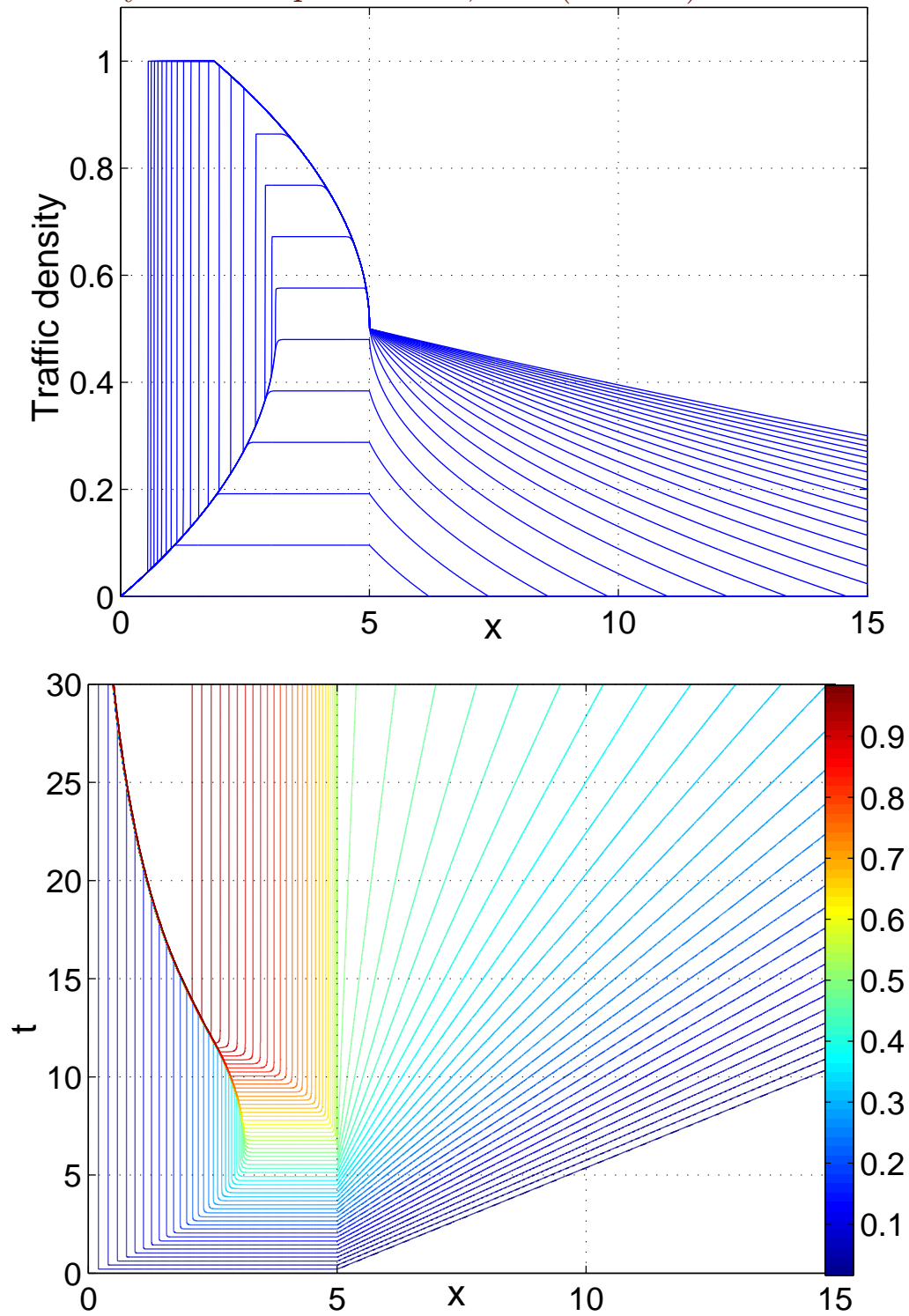
(a) entrances
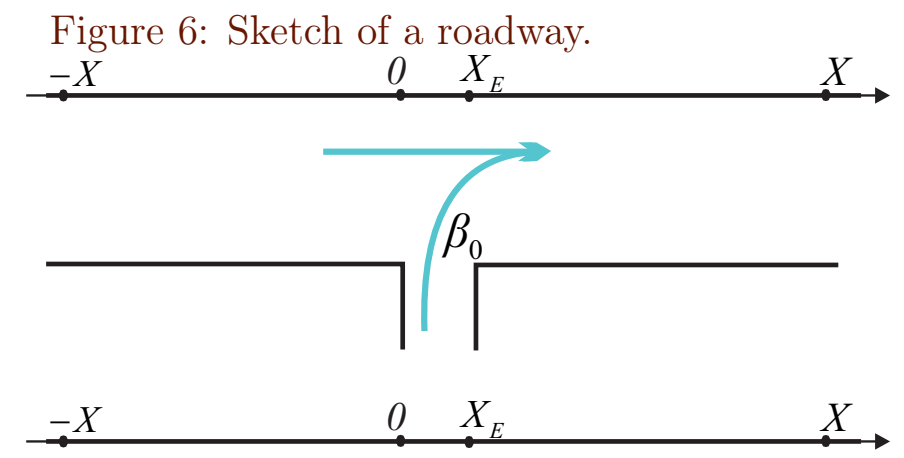

(b) exits

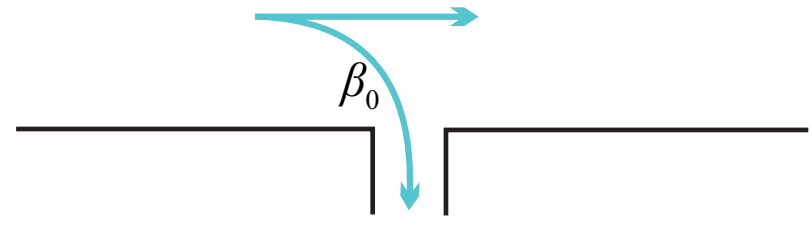

Simulations were conducted using $X=6$ and $X_{E}=0.2$, with $\Delta x=0.01$ and $\Delta t=\Delta x / V_{m}$ to ensure stability, and again adopting the normalized parameters $\mathrm{N}_{\mathrm{m}}=1$ and $\mathrm{V}_{\mathrm{m}}=1$. In the first case, the discrete model (3) is implemented using $\beta_{i}^{j}=0.6$ for all $x_{i} \in\left[0, X_{E}=0.2\right]$. This entrance gives a total entrance flux $f=0.6 X_{E}=0.12$ for each time unit. For the parabolic flux function (2), two traffic densities $n_{1}=0.1394$ and $n_{2}=0.8605$ correspond to this entrance flux, representing low and high densities, respectively. Starting with the initial traffic density $n(x, 0) / N_{m}=0.5$, the entrance induces an additional flux that evolves into a backward-propagating shock wave with speed determined by the Rankine-Hugoniot formula $\left(f\left(n_{2}\right)-f(0.5)\right) /\left(n_{2}-0.5\right)$. The effect of entrance rate $\beta_{i}^{j}=0.6$ for $x_{i} \in\left[0, X_{E}\right]$ to the initial density $n(x, 0) / N_{m}=0.5$ is shown on $n(x, 8) / N_{m}$ as plotted in Figure 7 (top). On the other hand, the effect of exit rate $\beta_{i}^{j}=-0.6$ for $x_{i} \in\left[0, X_{E}\right]$ given the initial density $n(x, 0) / N_{m}=0.5$ is to induce a forward-propagating shock wave as shown in Figure 7 (bottom). The speed of this forward propagating shock wave is determined by $\left(f\left(n_{1}\right)-f(0.5)\right) /\left(n_{1}-0.5\right)$. 
Figure 7: Traffic density $n / N_{m}$ after 800 time iteration, showing the effects of exits or entrances on the initial traffic density $n(x, 0) / \mathrm{N}_{m}=0.5$ (dash dot black line). Exit-entrance parameters; a constant rate $\beta_{i}^{j}=0.6$ (blue dashed curves), the Poisson distributed rate with the expected parameter $\mu=44$ vehicles per hour (red solid curves).
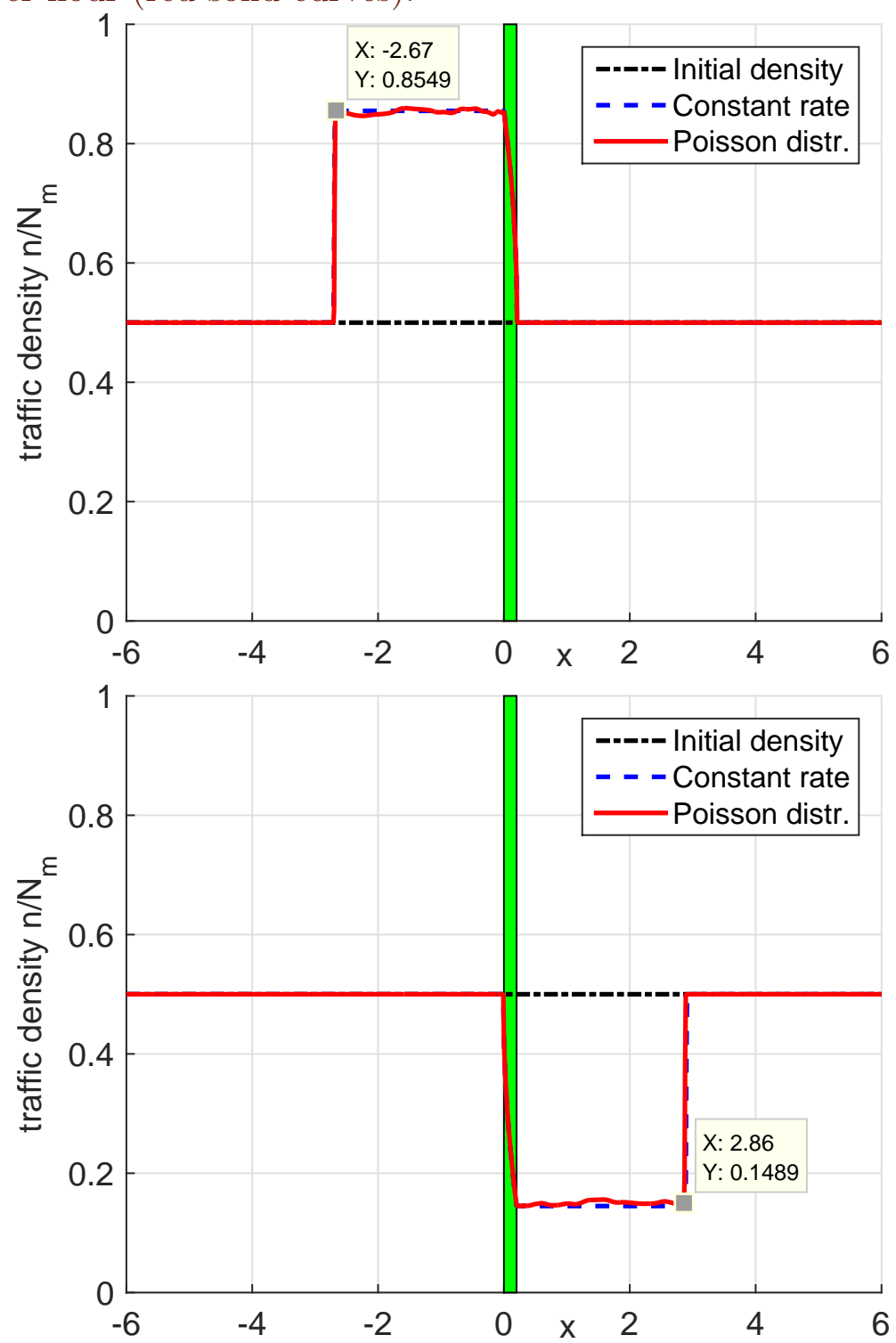
Figure 8: Traffic density $n / N_{m}$ after 800 time iteration, showing the effect of entrances on the initial density $\mathfrak{n}(\mathrm{x}, 0) / \mathrm{N}_{\mathrm{m}}=0.3$ (top), and exits on the initial density $\mathfrak{n}(x, 0) / \mathrm{N}_{\mathrm{m}}=0.7$ (bottom). Exit-entrance parameters; a constant rate $\beta_{i}^{j}=0.6$ (blue dashed curves), the Poisson distributed rate with the expected parameter $\mu=44$ vehicles per hour (red solid curves).
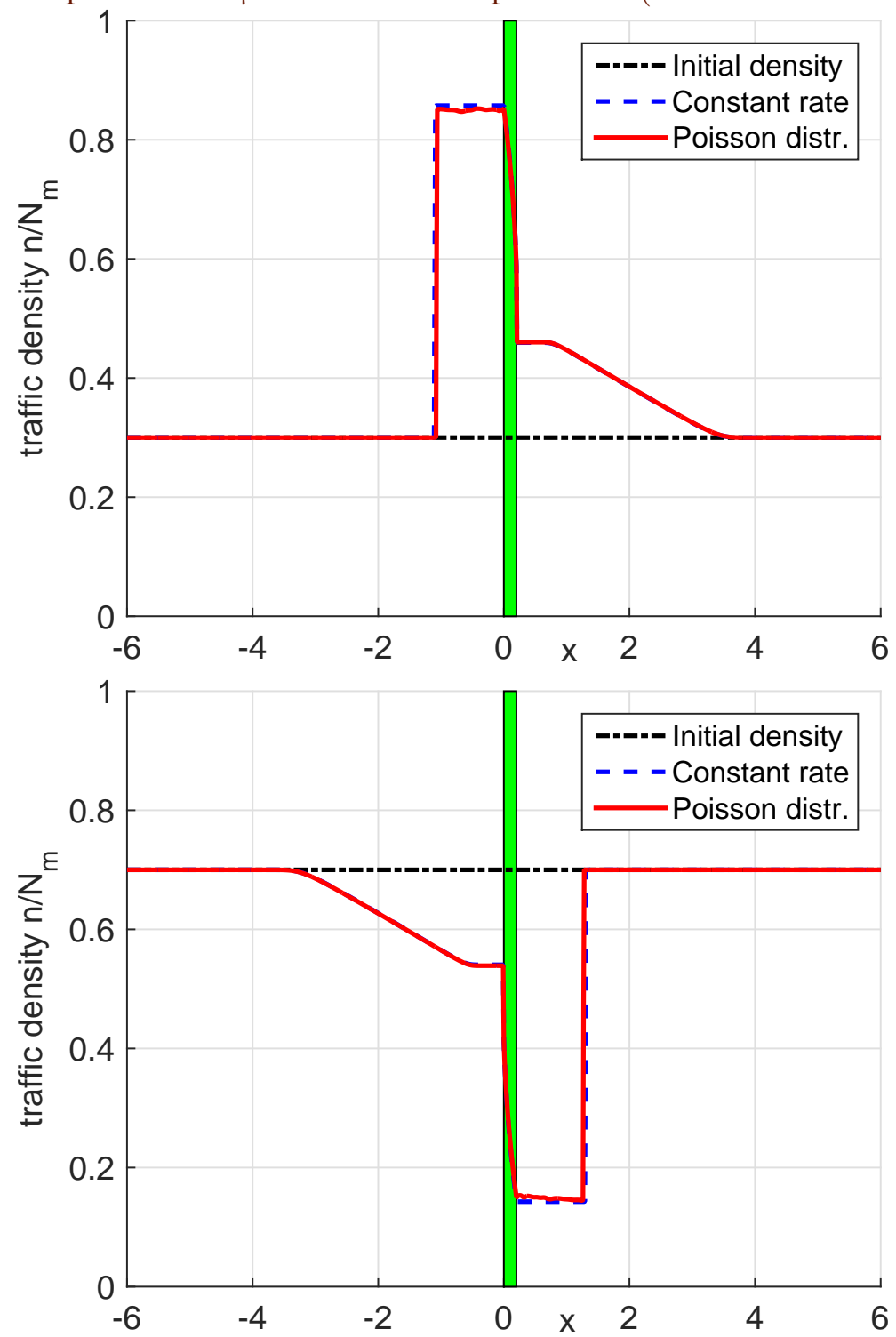
Table 1: Height and speed of the numerical shock wave of traffic density compare with the analytical prediction.

\begin{tabular}{lcrc}
\hline & Numeric & Analytic & error (\%) \\
\hline low density $\left(\mathrm{n}_{1}\right)$ & 0.1489 & 0.1394 & 0.6 \\
(forward) speed & $2.86 / 8=0.3575$ & 0.3606 & 0.8 \\
\hline high density $\left(\mathrm{n}_{2}\right)$ & 0.8549 & 0.8605 & 0.6 \\
(backward) speed & $\left(-2.67-\mathrm{X}_{\mathrm{E}}\right) / 8=-0.3587$ & -0.3605 & 0.5 \\
\hline
\end{tabular}

Further, we show that the shock wave in our simulations agree with the analytical prediction. Agreement is encountered on the shock wave height, as well as its speed. As shown in Figure 7 (bottom) the shock wave height is 0.1489 , which approximates the low density $\mathrm{n}_{1}$ to within $0.6 \%$ accuracy. Moreover, the shock wave front of $\eta(x, 8) / \mathrm{N}_{m}$ is located at $x=2.86$ which results in a shock wave speed $2.86 / 8=0.3575$. This numerical shock speed confirms the analytical shock speed $\left(f\left(n_{1}\right)-f(0.5)\right) /\left(n_{1}-0.5\right)=0.3606$ within $0.8 \%$ accuracy. Quantitative comparison is also conducted for the high density shock wave $n_{2}$. The results summarised in Table 1 show that the speed of traffic density propagation is computed accurately. Moreover, our simulation results show a good quantitative agreement with the analytical predictions with error less than $1 \%$.

Furthermore, when the initial density is taken to be $n(x, 0) / N_{m}=0.3$ (Figure 8, top), in addition to the backward propagating shock wave with height $n_{2}$, the entrances also induce a forward propagating rarefaction wave in the downstream area $x>X_{E}$. Another simulation using the initial traffic density $\mathfrak{n}(x, 0) / \mathrm{N}_{\mathrm{m}}=0.7$ with the same exit rate induces signals that propagate both to the right and to the left (Figure 8, bottom).

Similar results are obtained when the above computations are repeated using physical parameters $\mathrm{N}_{\mathrm{m}}=150$ vehicles $/ \mathrm{km}$ and $\mathrm{V}_{\mathrm{m}}=50 \mathrm{~km} /$ hour on the same computational domain with $X=6 \mathrm{~km}$ and $X_{E}=0.2 \mathrm{~km}$. Computations of (3) were conducted using $\Delta x=0.01$, where for stability reasons we set $\Delta \mathrm{t}=\Delta \mathrm{x} / \mathrm{V}_{\mathrm{m}}=0.01 / 50$ hour $=0.7 \mathrm{sec}$. Starting from the initial density 
$\mathrm{n}(x, 0) / \mathrm{N}_{\mathrm{m}}=0.5$, the same left-propagating shock wave appear in response to traffic entrances with $\beta_{i}^{j} \Delta t=0.6 \mathrm{~N}_{\mathrm{m}} / \mathrm{V}_{\mathrm{m}}=90 / \mathrm{V}_{\mathrm{m}}=1.8$ vehicles per cell per time iteration. The resulting traffic density after 800 time iterations, $n(x, t=0.16$ hour $) / N_{m}$, is exactly the same as the traffic density calculated using the normalized parameters, and in Figure 7 they are both plotted as a single blue dashed curve.

Next, we present another simulation using a Poisson-distributed entrance rate with the probability density function

$$
p(x)=\frac{\mu^{x} e^{-\mu}}{x !},
$$

with the expected value $\mu$ denoting the number of vehicles arriving in a given time interval. Comparable results to those of the deterministic case will be obtained if we choose $\mu=44$ vehicles per cell per time iteration, since the expected value $\mu$ should be close to the entrance rate of $0.6 \mathrm{~N}_{\mathrm{m}}=90$ vehicles per cell per time iteration. In Figure 7 , the solid red curve shows the traffic density after 800 time iterations, $\mathfrak{n}(x, t=0.16$ hour $) / N_{m}$, resulting from the Poisson-distributed exit-entrance rate.

Alike computations with identical physical parameters and Poisson-distributed entrance rates were conducted using the initial density $n(x, 0) / N_{m}=0.3$, and again the same phenomenon is observed (Figure 8, top): the entrance induces a backward-propagating shock wave and a forward-propagating rarefaction wave. Computation was repeated for exit with the same rate $\beta_{i}^{j}=-0.6$ using initial conditions $n(x, 0) / N_{m}=0.7$. The computed traffic density after 800 time iterations is plotted in Figure 8 (bottom). Based on these simulations, we conclude that the deterministic results are comparable with those obtained from the Poisson-distributed stochastic model; both exhibit similar characteristics of traffic congestion and diffusion. 


\subsection{More experiments with exits and entrances}

Various simulations were conducted to observe the effects of exits and entrances from two successive junctions. A computational domain $[-X, 2 X]$ is considered with two junctions located on $\left[0, X_{E}\right]$ and $\left[X, X+X_{E}\right]$ : in figures these areas are indicated in green. The same normalized parameters were used as in the previous simulations, but here we select $\beta_{i}^{j}=0.15$, and conduct computations using $X=6$ and $X_{E}=0.2$, and the result is given in Figure 9 (top). As time progresses, the initial traffic density $n(x, 0) / N_{m}=0.3$ changes due to entrances. This higher-density traffic continues to travel to the right until it reaches the second junction, from which points exits begin to occur with the same rate $\beta_{i}^{j}=-0.15$. The outcome is that the net traffic density on the downstream part of the domain $\left[X+X_{E}, 2 X\right]$ is nearly the same as the initial traffic density $\mathfrak{n}(\boldsymbol{x}, 0) / \mathbf{N}_{\mathrm{m}}$, which is to be expected. Figure 9 (bottom) presents a similar result, but now starting from an initial traffic density $n(x, 0) / N_{m}=0.7$, and traffic exits from the first junction and enters at the second junction with the same rate. Simulation result shows that the low exit rate on high initial density only affects the upstream part, leaving the downstream part unchanged, as expected. For the two cases above, computations were also conducted using Poisson-distributed exit and entrance rates, and both results show a good match with those of constant rate exit entrance cases.

Figures 10 and 11 present another simulation but using a higher exit entrance rate. In Figure 10 (top) a high entrance rate $\beta_{i}^{j}=0.6$ on $\left[X, X+X_{E}\right]$ induces a high traffic density in the form of a shock wave that propagates to the left, as well as a rarefaction wave that propagates to the right. Once the rarefaction wave arrives at the second junction $\left[X, X+X_{E}\right]$, traffic exit with the same rate $\beta_{i}^{j}=-0.6$ so that traffic density back to the initial value $\mathfrak{n}(x, 0) / N_{m}=0.3$. Figure 10 (bottom) presents the other way around; exit followed by an entrance of the same rate, conducted using an initial traffic density $n(x, 0) / N_{m}=0.7$. Here the low density propagates into the middle part of the domain, whereas a rarefaction wave propagates to the left. 
Figure 9: Snapshots of traffic density using $\beta= \pm 0.15$ at subsequent times after 100, 500 time iterations, and final time iteration 2000. Changes of traffic density are due to entrances on $\left[0, X_{E}\right]$ and later exits on $\left[X, X+X_{E}\right]$ (top), and due to exits on $\left[0, X_{E}\right]$ and later entrances on $\left[X, X+X_{E}\right]$ (bottom).
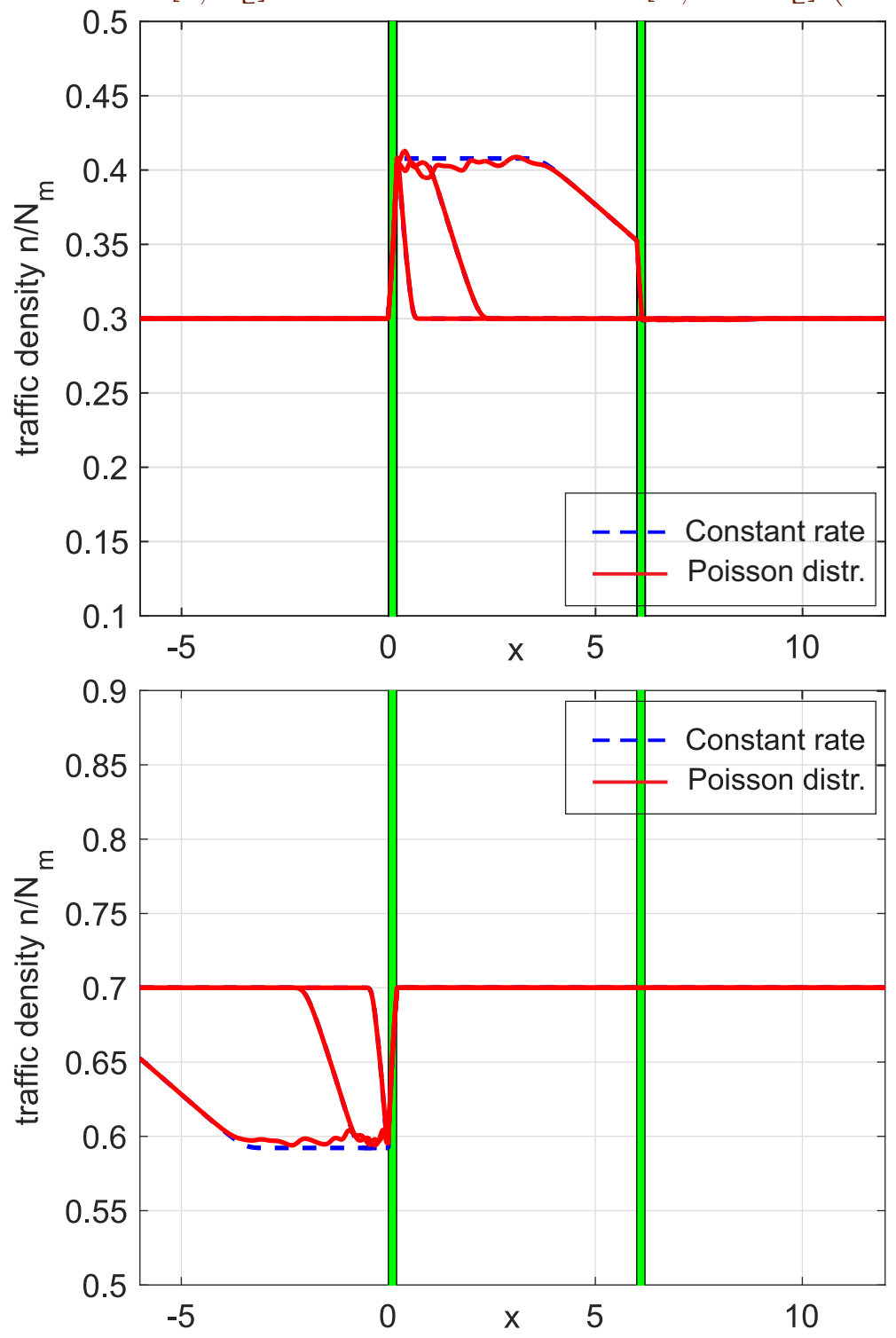
Figure 10: Snapshots of traffic density using $\beta= \pm 0.6$ at subsequent times (after 100, 500 and 2000 time iterations); (top) due to entrances on $\left[0, X_{\mathrm{E}}\right]$ and later exits on $\left[X, X+X_{E}\right]$, (bottom) due to exits on $\left[0, X_{E}\right]$ and later entrances on $\left[X, X+X_{E}\right]$.
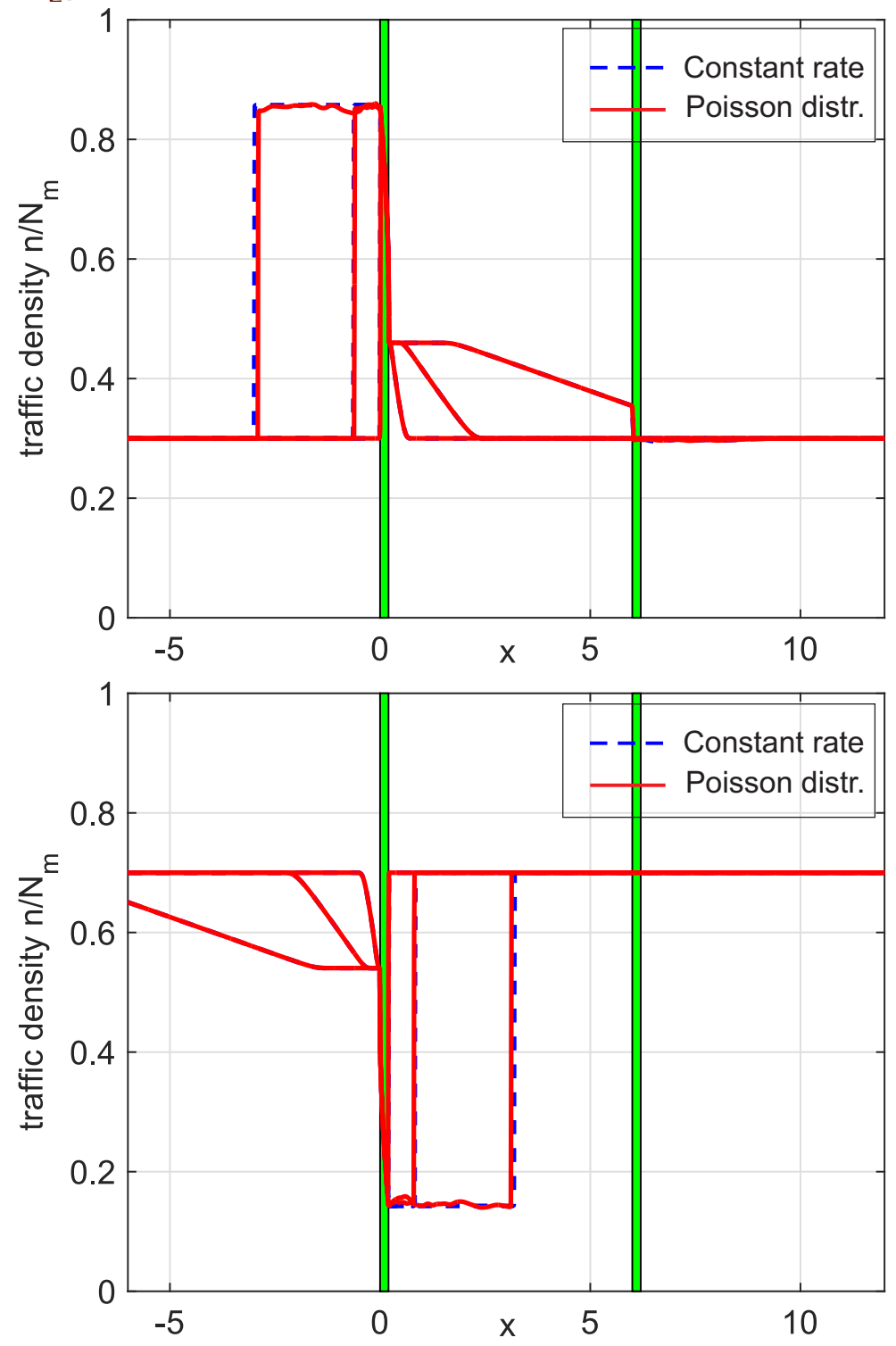
Figure 11 presents similar phenomena as the outcome of simulation using much higher exit entrance rate $\beta_{i}^{j}=1.5$. In these simulations, the stop and go procedure (5)-(6) is crucial to ensure the computed traffic density $n(x, t) / N_{m}$ remains between zero and one. All simulations above were calculated using both a constant rate and a Poisson-distributed exit and entrance rates. The good agreement between both approaches support the idea that a deterministic approach can be used for analysing stochastic traffic dynamics problems.

\section{Conclusions}

We proposed a conservative finite volume scheme with an upwind approximation for the flux function to solve the kinematic LWR model with a source term. A kind of stop-go procedure was successfully implemented in order to restrict traffic density values to fall between zero and the maximum-capacity value in the simulations. The numerical method is stable, and the traffic densities which evolve in response to a light (and heavy) entrance rates are shown to conform closely to previous analytical results. In addition, for a long time simulation, compliance with analytical formula were obtained for the height and speed of the shock wave of traffic density. From further simulations involving exits and entrances, we found that in low traffic density, a low entrance rate would induce a right propagated rarefaction wave, whereas a high entrance rate would induce an additional left propagating shock wave. In high traffic density, a low exit rate would induce a left propagated rarefaction wave, whereas a high exit rate would induce an additional right propagating shock wave. The above results hold for simulations conducted using constant exit/entrance rates as well as Poisson-distributed rates.

Acknowledgement SRP acknowledges the financial support from ITB Research Grant 2018. We sincerely thank Andrew Schauf for improving the writing of this manuscript. 
Figure 11: Snapshots of traffic density using $\beta= \pm 1.5$ at subsequent times (after 100, 500 and 2000 time iterations); (top) due to entrances on $\left[0, X_{\mathrm{E}}\right]$ and later exits on $\left[X, X+X_{E}\right]$, (bottom) due to exits on $\left[0, X_{E}\right]$ and later entrances on $\left[X, X+X_{E}\right]$.
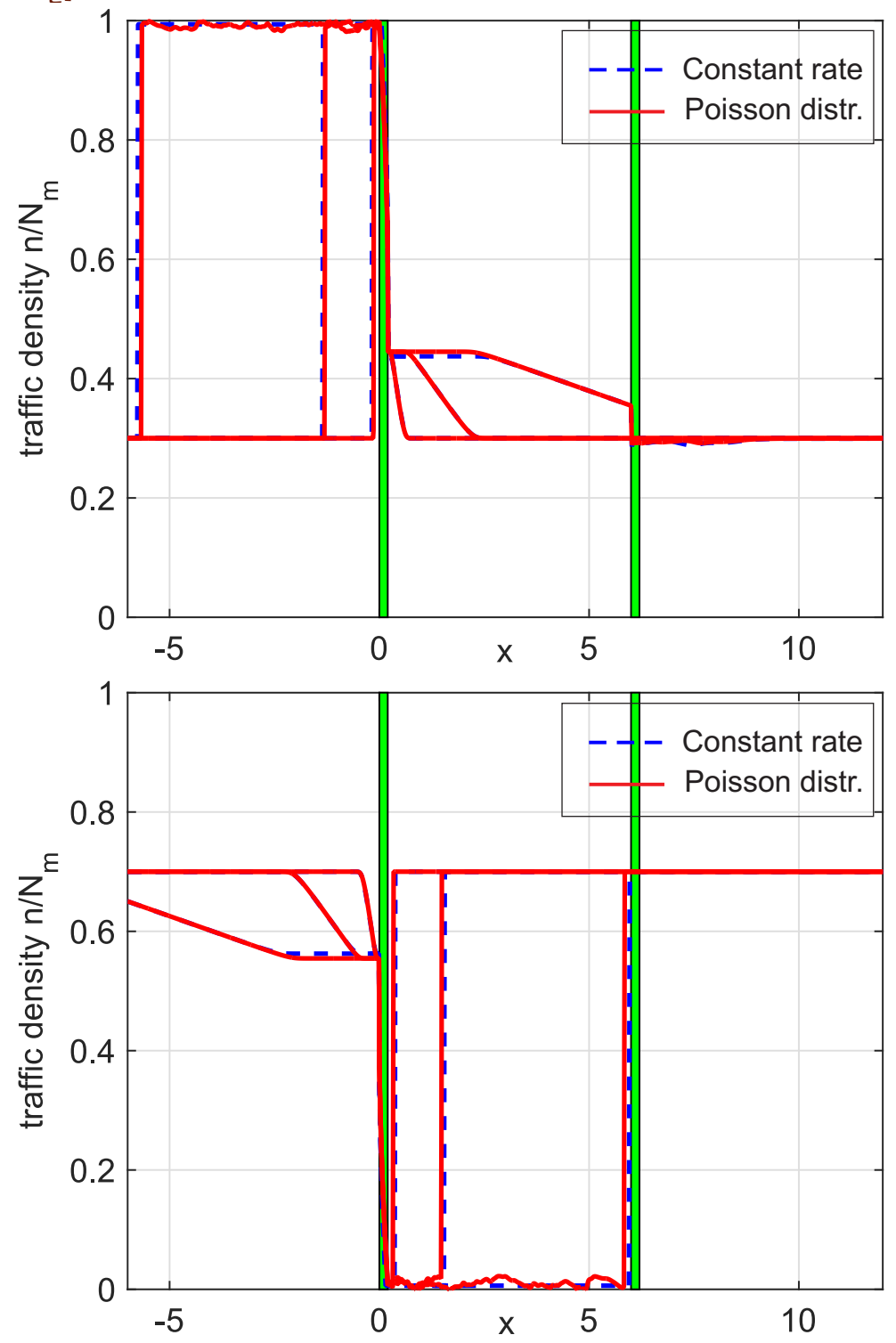


\section{References}

[1] P. Bagnerini, R. M. Colombo, A. Corli, S. Pedretti, Conservation Versus Balance Laws in Traffic Flow, Traffic and Granular Flow '03, 235-240, (2005). doi:10.1007/3-540-28091-X_22 E3

[2] C. F. Daganzo, The cell transmission model: a dynamic representation of highway traffic consistent with hydrodynamic theory, Transportation Research B, 28(4), 269-287 (1994). doi:10.1016/0191-2615(94)90002-7 E3

[3] C. F. Daganzo, The cell transmission model II: Network traffic, Transportation Research B, 29(2), 79-93, (1995). doi:10.1016/0191-2615(94)00022-R E3

[4] N. D. Fowkes, J. J. Mahony, An Introduction to Mathematical Modelling. John Wiley \& Sons, England, (1994). E3

[5] R. Haberman, Mathematical Model, Prentice-Hall, Inc., New York, (1977). doi:10.1137/1.9781611971156 E3, E7

[6] H. Holden and N. H. Risebro. A mathematical model of traffic flow on a network of unidirectional roads, SIAM Journal on Mathematical Analysis, 26(4):999-1017, (1995). doi:10.1137/S0036141093243289 E3

[7] J. P. Lebacque, The godunov scheme and what it means for first order traffic flow models, In The International Symposium on Transportation and Traffic Theory, Lyon, France, (1996).

http://worldcat.org/isbn/0080425860 E3

[8] R. M. M. Mattheij, ten Thije Boonkkamp, S. W. Rienstra, Partial Differential Equation: Modelling, Analysis, Computation, The Netherlands Society for Industrial and Applied Mathematics, Philadelphia, (2005). doi:10.1137/1.9780898718270 E3, E6 
[9] M. Mercier, Traffic flow modelling with junctions, Jour. of Math. Analysis and Applications, 350 (1), 369-383, (2009). doi:10.1016/j.jmaa.2008.09.040 E3

[10] G. Orosz, R. E. Wilson, G. Stépána, Traffic jams: dynamics and control, Phil. Trans. R. Soc. A 368, 2010, pp. 4455-4479. doi:10.1098/rsta.2010.0205 E3

[11] M. Papageorgiou, Dynamic modelling, assignment and route guidance in traffic networks, Transportation Research B, 24(6), (1990), pp. 471-495. doi:10.1016/0191-2615(90)90041-V E3

[12] S.R. Pudjaprasetya, J. Bunawan, C. Novtiar, Traffic light or roundabout? Analysis using the modified kinematic LWR model, East Asian Journal of Applied Mathematics, 6 (1), 80-88, (2016). doi:10.4208/eajam.210815.281215a E6

\section{Author addresses}

1. S. R. Pudjaprasetya, Industrial and Financial Mathematics Research Group, Faculty of Mathematics and Natural Sciences, Institut Teknologi Bandung, Jalan Ganesha 10, Bandung 40132, Indonesia. mailto:sr_pudjap@math.itb.ac.id orcid:0000-0001-6346-0272

2. P. Z. Kamalia, Industrial and Financial Mathematics Research Group, Faculty of Mathematics and Natural Sciences, Institut Teknologi Bandung, Jalan Ganesha 10, Bandung 40132, Indonesia. orcid:0000-0003-1938-0368 